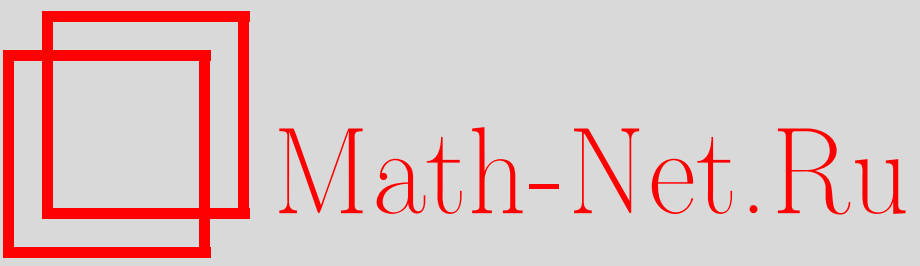

Р. Р. Булатова, Нестационарный пограничный слой модифицированной вязкой жидкости, Итоги науки и техн. Сер. Соврем. мат. и ее прил. Темат. обз., 2021, том 191, 10-15

DOI: https://doi.org/10.36535/0233-6723-2021-191-10-15

Использование Общероссийского математического портала Math-Net.Ru подразумевает, что вы прочитали и согласны с пользовательским соглашением

http://www. mathnet.ru/rus/agreement

Параметры загрузки:

IP : 35.173 .219 .149

26 апреля 2023 г., 14:29:04 
ИТОГИ НАУКИ И ТЕХНИКИ.

Современная математика и ее приложения.

Тематические обзоры.

Том 191 (2021). C. 10-15

DOI: 10.36535/0233-6723-2021-191-10-15

УДК 517.956 .45

\title{
НЕСТАЦИОНАРНЫЙ ПОГРАНИЧНЫЙ СЛОЙ МОДИФИЦИРОВАННОЙ ВЯЗКОЙ ЖИДКОСТИ
}

\author{
(c) 2021 г. $\quad$ P. Р. БУЛАТОВА
}

\begin{abstract}
АннотАция. Изучается система уравнений нестационарного симметричного пограничного слоя нелинейно вязкой несжимаемой жидкости. С помощью преобразования Крокко система пограничного слоя сводится к одному квазилинейному вырождающемуся параболическому уравнению. Доказана однозначная разрешимость основной краевой задачи.
\end{abstract}

Ключевъе слова: пограничный слой, нестационарный поток, переменные Крокко, модифицированная жидкость Ладыженской.

\section{UNSTEADY BOUNDARY LAYER OF A MODIFIED VISCOUS FLUID}

\author{
(C) 2021 R. R. BULATOVA
}

\begin{abstract}
In this paper, a system of equations for a nonstationary, symmetric boundary layer of a nonlinearly viscous, incompressible fluid is studied. By using the Crocco transformation, we reduce the boundary-layer system to a single quasilinear degenerate parabolic equation. The unique solvability of the main boundary-value problem is proved.
\end{abstract}

Keywords and phrases: boundary layer, unsteady flow, Crocco variables, modified Ladyzhenskaya fluid.

AMS Subject Classification: 34B15

1. Введение. Теория пограничного слоя, изучающая движение вязких сплошных сред в окрестности твердой поверхности, составляет один из важнейших разделов гидродинамики. Её основателем является Л. Прандтль, предложивший для описания динамики маловязкой жидкости вблизи обтекаемой твердой поверхности систему уравнений, которая при определенных предположениях выводится из системы Навье-Стокса и является ее упрощением. На основе аксиом Прандтля для неньютоновских жидкостей можно также вывести систему уравнений, обобщающую классическую систему пограничного слоя, которая применяется для описания движения вязких сред со сложной реологией. О корректной разрешимости основных краевых и начальнокраевых задач для системы уравнений Прандтля (см. [2]).

Позже О. А. Ладыженская предложила некоторую модификацию системы уравнений НавьеСтокса с целью получить корректную разрешимость основных краевых задач для уравнений гидродинамики в трехмерном случае. В [3] из модифицированной системы уравнений выведена соответствующая система уравнений пограничного слоя и доказана однозначная разрешимость основной граничной задачи для нее. Изучение системы пограничного слоя в электромагнитной среде было продолжено в ряде более поздних работ (см. [1,5]).

В данной работе изучается задача о пограничном слое, возникающем при обтекании симметричного тела нестационарным потоком вязкой несжимаемой жидкости Ладыженской. Основным 
методом решения поставленных задач является преобразование Крокко, которое переводит нестационарную систему уравнений пограничного слоя в одно квазилинейное уравнение. Решение задачи получено в некоторой окрестности критической точки (см. [4]). Задача о нестационарном симметричном слое решена в целом по $t$. При этом решения рассматриваются в классе функций, имеющих асимптотику вида $u(t, x, y)-U(t, x) \sim \exp \left\{-M y^{2}\right\}$ при $y \rightarrow \infty$.

2. Преобразование Крокко. Рассмотрим случай двумерного нестационарного течения модифицированной системы уравнений пограничного слоя:

$$
\nu\left(1+3 d\left(u_{y}\right)^{2}\right) u_{y y}-u_{t}-u u_{x}-v u_{y}=-U_{t}-U U_{x}, \quad u_{x}+v_{y}=0 .
$$

Здесь $\nu, d$-постоянные, зависящие от свойств жидкости, плотность жидкости $\rho$ предполагается равной единице, $U(t, x)$ - заданная функция, связанная с давлением $p(t, x)$ уравнение Эйлера для скорости внешнего потока

$$
-p_{x}=U_{t}+U U_{x}
$$

Система уравнений (1) рассматривается в области $D=\{0<t<\infty, 0<x<X, 0<y<\infty\}$ с начальным и граничными условиями

$$
\begin{gathered}
u(0, x, y)=u_{0}(x, y), \quad u(t, 0, y)=0, \quad u(t, x, 0)=0, \\
v(t, x, 0)=v_{0}(t, x), \quad u(t, x, y) \rightarrow U(t, x) \text { при } y \rightarrow \infty,
\end{gathered}
$$

где $U(t, 0)=0, U_{x}(t, 0)>0, U(t, x)>0$ при $x>0$, а функция $v_{0}(t, x)$ предполагается заданной. Условия $U(t, 0)=0$ и $u(t, 0, y)=0$ определяют точку $x=0$ как точку, в которой происходит остановка внешнего потока жидкости и пограничный слой симметричен относительно этой точки. Дополнительно предположим, что $U(t, x)=x V(t, x), V(t, x)>0$ и $V, V_{x}, V_{t}, v_{0}$ ограничены при $0<x \leqslant X$.

Определение 1. Решением задачи (1), (2) называется пара функций $u(t, x, y)$ и $v(t, x, y)$, обладающая следующими свойствами: $u(t, x, y)$ непрерывна и ограничена в замкнутой области $\bar{D}$, $v(t, x, y)$ непрерывна по $y$ в $\bar{D}$ и ограничена при ограниченных $y$; обобщенные производные $u_{t}, u_{x}$, $u_{y}, u_{y y}, v_{y}$ являются ограниченными измеримыми функциями; $u$ и $v$ удовлетворяют системе $(1)$ в $D$ и граничным условиям (2).

Задачу (1), (2) сведем к некоторой вспомогательной краевой задаче для одного квазилинейного уравнения. Для этого введем новые независимые переменные $\tau, \xi, \eta$ и новую неизвестную функцию $w(\tau, \xi, \eta)$ следующим образом:

$$
\tau=t, \quad \xi=x, \quad \eta=\frac{u(t, x, y)}{U(t, x)}, \quad w(\tau, \xi, \eta)=\frac{u_{y}(t, x, y)}{U(t, x)} .
$$

Приведем систему (1) к одному квазилинейному уравнению. Исключим $v$ из системы уравнений (1), продифференцировав эти уравнения по у. Имеем

$$
\nu\left(1+3 d\left(u_{y}\right)^{2}\right) u_{y y y}+6 \nu d u_{y y}^{2} u_{y}-u_{t y}-u_{y} u_{x}-u u_{x y}-v_{y} u_{y}-v u_{y y}=0 .
$$

Из системы уравнений (1) находим

$$
v=\frac{1}{u_{y}}\left(\nu\left(1+3 d\left(u_{y}\right)^{2}\right) u_{y y}-u_{t}-u u_{x}+U_{t}+U U_{x}\right), \quad v_{y}=-u_{x} .
$$

Как следствие этих трех равенств, выводим

$$
\begin{aligned}
0=\nu\left(1+3 d\left(u_{y}\right)^{2}\right) u_{y y y}-\nu\left(1+3 d\left(u_{y}\right)^{2}\right) \frac{u_{y y}^{2}}{u_{y}}+\nu u_{y y}^{2} 6 d u_{y}-u_{t y}+u_{t} \frac{u_{y y}}{u_{y}}- \\
-\left(u u_{x y}-\frac{u_{y y}}{u_{y}} u u_{x}\right)-U U_{x} \frac{u_{y y}}{u_{y}}-U_{t} \frac{u_{y y}}{u_{y}}=\nu\left(1+3 d\left(u_{y}\right)^{2}\right) \frac{u_{y y y} u_{y}-u_{y y}^{2}}{u_{y}}- \\
-u \frac{u_{x y} u_{y}-u_{y y} u_{x}}{u_{y}}-\frac{u_{y y}}{u_{y}} U U_{x}+6 \nu d u_{y} u_{y y}^{2}-u_{t y}+u_{t} \frac{u_{y y}}{u_{y}}-U_{t} \frac{u_{y y}}{u_{y}}
\end{aligned}
$$


Вернемся к соотношениям (3), из которых получим

$$
\begin{gathered}
u=\eta U, \quad u_{y}=w U, \quad x=\xi \\
u_{y y}=w_{\eta} \eta_{y} U=w_{\eta} u_{y}, \quad \frac{u_{y y}}{u_{y}}=w_{\eta}, \\
u_{y y y}=w_{\eta \eta} \eta_{y} u_{y}+w_{\eta} u_{y y}=w_{\eta \eta} \frac{u_{y}^{2}}{U}+w_{\eta} u_{y y}, \\
u_{y x}=w U_{\xi}+U w_{\xi}+w_{\eta} u_{\xi}-w_{\eta} u \frac{U_{\xi}}{U}, \quad u_{y} u_{y y}^{2}=w_{\eta}^{2} w^{3} U^{3}, \\
\frac{u_{x y} u_{y}-u_{x} u_{y y}}{u_{y}}=w_{\xi} U+w U_{\xi}-w_{\eta} u_{\xi}+w_{\eta} u_{\xi}-w_{\eta} \eta U_{\xi}, \\
\frac{u_{y y y} u_{y}-u_{y y}^{2}}{u_{y}}=w_{\eta \eta} \frac{u_{y}^{2}}{U}+w_{\eta} u_{y y}-\frac{u_{y y}}{u_{y}} u_{y y}=w_{\eta \eta} \frac{u_{y}^{2}}{U}+w_{\eta} u_{y y}-w_{\eta} u_{y y}=w_{\eta \eta} \frac{u_{y}^{2}}{U^{2}} U=w_{\eta \eta} w^{2} U, \\
=-\eta w_{\xi} U-\eta w U_{\xi}+\eta^{2} w_{\eta} U_{\xi}-w_{\eta} U_{\xi}=\left(\eta^{2}-1\right) w_{\eta} U_{\xi}-\eta w_{\xi} U-\eta w U_{\xi}, \\
\left.u \frac{u_{x y} u_{y}-u_{x} u_{y y}}{u_{y}}+\frac{u_{y y}}{u_{y}} U U_{x}\right)=-\frac{u}{U}\left(w_{\xi} U+w U_{\xi}-w_{\eta} \eta U_{\xi}\right)-w_{\eta} U_{\xi}= \\
u_{t y}=\left(w_{\tau} \tau_{t}+w_{\eta} \eta_{t}\right) U+w U_{t}=w_{\tau} U+\eta_{t} w_{\eta} U+w U_{t}=w_{\tau} U+\frac{u_{t} U-u U_{t}}{U^{2}} w_{\eta} U+w U_{t}= \\
=w_{\tau} U+w U_{t}+w_{\eta} u_{t}-\eta w_{\eta} U_{t}, \\
\frac{1}{U}\left(-u_{t y}+u_{t} \frac{u_{y y}}{u_{y}}-U_{t} \frac{u_{y y}}{u_{y}}\right)=\frac{1}{U}\left(-w_{\tau} U-w U_{t}-u_{t} w_{\eta}+\eta U_{t} w_{\eta}+u_{t} w_{\eta}-U_{t} w_{\eta}\right)= \\
=(\eta-1) \frac{U_{t}}{U} w_{\eta}-\frac{U_{t}}{U} w-w_{\tau} .
\end{gathered}
$$

Преобразуем граничные условия:

$$
\begin{gathered}
v u_{y}=-u_{t}-u u_{x}+\nu\left(1+3 d\left(u_{y}\right)^{2}\right) u_{y y}+U_{t}+U U_{x}, \\
v \frac{u_{y}}{U}=-\frac{u_{t}}{U}-u \frac{u_{x}}{U}+\nu\left(1+3 d\left(u_{y}\right)^{2}\right) \frac{u_{y y}}{U}+\frac{U_{t}}{U}+U_{x}, \\
v \frac{u_{y}}{U}=-\frac{u_{t}}{U}-u \frac{u_{x}}{U}+\nu\left(\frac{u_{y}}{U}+3 d \frac{u_{y}^{3}}{U^{3}} U^{2}\right) \frac{u_{y y}}{u_{y}}+\frac{U_{t}}{U}+U_{x}, \\
v w=-\frac{u_{t}}{U}-u \frac{u_{\xi}}{U}+\nu\left(w+3 d U^{2} w^{3}\right) w_{\eta}+\frac{U_{t}}{U}+U_{x} .
\end{gathered}
$$

Легко видеть, что $u(t, x, 0)=0$ и, следовательно, из $y=0$ вытекает $\eta=0$. Таким образом, из условия $y=0$, следует

$$
\nu\left(1+3 d U^{2} w^{2}\right) w w_{\eta}-v_{0} w+\frac{U_{t}}{U}+U_{x}=0 .
$$

В итоге, учитывая (2), выводим одно квазилинейное уравнение

$$
\begin{aligned}
\nu\left(1+3 d U^{2} w^{2}\right) w^{2} w_{\eta \eta}-w_{\tau}-\eta U w_{\xi}+\left(\eta^{2}-1\right) U_{x} w_{\eta} & +(\eta-1) \frac{U_{t}}{U} w_{\eta}- \\
& -\eta U_{x} w+6 \nu d U^{2} w_{\eta}^{2} w^{3}-\frac{U_{t}}{U} w=0
\end{aligned}
$$

в области $\Omega=\{0<\tau<\infty, 0<\xi<X, 0<\eta<1\}$ с условиями

$$
\begin{gathered}
w(\tau, \xi, 1)=0,\left.\quad\left(\nu\left(1+3 d U^{2} w^{2}\right) w w_{\eta}-v_{0} w+\frac{U_{t}}{U}+U_{x}\right)\right|_{\eta=0}=0 \\
w(0, \xi, \eta)=w_{0}(\xi, \eta)
\end{gathered}
$$


где

$$
w_{0}(\xi, \eta)=\frac{u_{0 y}(x, y)}{U(0, x)}
$$

Определение 2. Функция $w(\tau, \xi, \eta)$ называется решением задачи $(4),(5)$, если она непрерывна в $\bar{\Omega}$ и имеет ограниченные обобщенные производные $w_{\tau}, w_{\xi}, w_{\eta}$, причем $w_{\eta}$ непрерывна по $\eta$ при $\eta=0$; существует такая обобщенная производная $w_{\eta \eta}$, что произведение $w w_{\eta}$ ограничено в $\bar{\Omega} ;$ уравнение (4) выполняется для $w$ почти всюду в $\Omega$ и $w$ удовлетворяет условиям (5).

Далее воспользуемся введенным ранее предположением $U=x V$ и для удобства примем обозначения:

$$
A=\left(\eta^{2}-1\right)\left(V+\xi V_{x}\right)+(\eta-1) \frac{V_{t}}{V}, \quad B=-\eta\left(V+\xi V_{x}\right)-\frac{V_{t}}{V}, \quad C=V+\xi V_{x}+\frac{V_{t}}{V} .
$$

Тогда задача (4) примет вид

$$
\nu\left(1+3 d \xi^{2} V^{2} w^{2}\right) w^{2} w_{\eta \eta}-w_{\tau}-\eta \xi V w_{\xi}+A w_{\eta}+B w+6 \nu d \xi^{2} V^{2} w^{3} w_{\eta}^{2}=0,
$$

а ее начальное и граничные условия (5) - вид

$$
\begin{gathered}
w(\tau, \xi, 1)=0,\left.\quad\left(\nu\left(1+3 d \xi^{2} V^{2} w^{2}\right) w w_{\eta}-v_{0} w+C\right)\right|_{\eta=0}=0, \\
w(0, \xi, \eta)=w_{0}(\xi, \eta) .
\end{gathered}
$$

Рассмотрим вспомогательную граничную задачу, решение которой будем использовать для оценок решений искомой задачи, а именно, рассмотрим дифференциальное уравнение

$$
L(Y) \equiv \nu Y^{2} Y_{\eta \eta}+\left(\eta^{2}-1\right) a Y_{\eta}-\eta a Y=0, \quad 0<\eta<1
$$

с граничными условиями

$$
\left.l(Y) \equiv\left(\nu Y Y_{\eta}-b Y+a\right)\right|_{\eta=0}=0, \quad Y(1)=0,
$$

где $a>0, b$ - некоторые константы. Всюду $C_{i}, M_{i}$ - положительные постоянные.

3. Основные утверждения. Имеют место следующие утверждения.

Теорема 1. Пусть $U_{x}>0$ при $0 \leqslant x \leqslant X ;$ функции $V, V_{x}, v_{0}, v_{0 x}, A_{x}, B_{x}, C_{x}$ ограничены;

$$
\begin{gathered}
\left|V_{t}\right| \leqslant M_{8} x, \quad\left|A_{t}\right| \leqslant M_{9} x, \quad\left|B_{t}\right| \leqslant M_{10} x, \quad\left|C_{t}\right| \leqslant M_{11} x, \quad\left|v_{0 t}\right| \geqslant-M_{12} x, \\
Y e^{-M_{1} \xi} \leqslant w_{0} \leqslant Y e^{M_{2} \xi}, \quad\left|w_{0 \xi}\right| \leqslant M_{3}(1-\eta)
\end{gathered}
$$

$w_{0}$ непрерывно дифберениируема по $\eta \in[0,1)$ u

$$
\begin{gathered}
Y_{\eta} e^{M_{4} \xi} \leqslant w_{0 \eta} \leqslant Y_{\eta} e^{-M_{5} \xi}, \\
-M_{6}(1-\eta) \leqslant \nu\left(1+3 d \xi^{2} V^{2} w_{0}^{2}\right) w_{0}^{2} w_{0 \eta \eta}+A w_{0 \eta}+B w_{0}+6 \nu d \xi^{2} V^{2} w_{0}^{3} w_{0 \eta}^{2} \leqslant M_{7} x(1-\eta), \\
\left.\left(\nu\left(1+3 d \xi V^{2} w_{0}^{2}\right) w_{0} w_{0 \eta}-v_{0} w_{0}+U_{x}+\frac{U_{t}}{U}\right)\right|_{\eta=0, \tau=0}=0 .
\end{gathered}
$$

Тогда задача (4), (5) в области $\Omega$ при $X$, зависящем от $V, v_{0}, w_{0}$ имеет решение $w(\tau, \xi, \eta)$, обладающее следующими свойствами: $w$ непрерьвна в $\bar{\Omega}$,

$$
Y e^{-C_{1} \xi} \leqslant w \leqslant Y e^{C_{2} \xi}
$$

$w_{\eta}$ непрерывна по $\eta<1$,

$$
Y_{\eta} e^{C_{3} \xi} \leqslant w_{\eta} \leqslant Y_{\eta} e^{-C_{3} \xi}
$$

обобщенные производные $w_{\tau}, w_{\xi}, w_{\eta \eta}$ удовлетворяют неравенствам

$$
-C_{5}(1-\eta) \leqslant w_{\tau} \leqslant C_{6} \xi(1-\eta), \quad\left|w_{\xi}\right| \leqslant C_{7} Y, \quad-C_{8} \leqslant w^{2} w_{\eta \eta} \leqslant-C_{9} .
$$

Решение задачи с такими свойствами единственно.

Обращая преобразование переменных (3), что возможно в силу свойств решения задачи (4), (5), получаем основной результат о существовании и единственности классического решения задачи (1), (2) в смысле данного нами определения. 
Теорема 2. Предположим, что

$$
U(t, x)=x\left(a+x a_{1}(t, x)\right), \quad v_{0}(t, x)=b+x b_{1}(t, x),
$$

где $a=$ const $>0, b=$ const; $U(t, x)>0$ при $x>0 ; a_{1}(t, x), a_{1 x}(t, x), a_{1 x x}(t, x), b_{1}(t, x), b_{1 x}(t, x)$ ограничены при $0 \leqslant t<\infty, 0 \leqslant x \leqslant X$. Пусть $u_{0}(x, y)$ удовлетворяет следующим условиям: функиия $u_{0} / U$ непрерывна в $\bar{D}, u_{0}>0$ при $y>0, x>0 ; u_{0}(x, 0)=0, u_{0} / U \rightarrow 1$ при $y \rightarrow \infty$; $u_{0 y} / U>0$ при $y \geqslant 0 ; u_{0 y} / U \rightarrow 0$ при $y \rightarrow \infty ;$ кроме того,

$$
\begin{gathered}
U(x) Y\left(\frac{u}{U}\right) e^{-M_{1} x} \leqslant u_{0 y} \leqslant U(x) Y\left(\frac{u}{U}\right) e^{M_{2} x}, \\
Y_{\eta}\left(\frac{u}{U}\right) e^{M_{4} x} \leqslant \frac{u_{0 y y}}{u_{0 y}} \leqslant Y_{\eta}\left(\frac{u}{U}\right) e^{-M_{5} x},
\end{gathered}
$$

Пусть выполнены условия согласования

$$
v_{0}(0, x) u_{0 y}(x, 0)=U_{t}(0, x)+U(0, x) U_{x}(0, x)+\nu\left(1+3 d\left(u_{0 y}\right)^{2}\right) u_{0 y y} .
$$

Тогда при некотором $X$, зависящем от функиий $U, u_{0}, v_{0}$, задача (1), (2) в области $D$ имеет единственное решение $u, v$, которое обладает следующими свойствами: $u>0$ при y $>0$ и $x>0$; функции и/U, uy $/ U$ ограничены и непрерывны в $\bar{D} ; u_{y}>0$ при $y \geqslant 0 ; u \rightarrow U$ при $y \rightarrow \infty, u_{y} / U \rightarrow 0$ при $y \rightarrow \infty$; функции $u_{x}, u_{y}, u_{y y}, u_{t}, v_{y}$ ограничены и непрерывны в $D$; функияя $v$ непрерывна в $\bar{D}$ по у и ограничена при ограниченных $y$; уравнения (1) выполняются почти всюду в $D$; имеют место оченки

$$
\begin{gathered}
U(x) Y\left(\frac{u}{U}\right) e^{-C_{1} x} \leqslant u_{y} \leqslant U(x) Y\left(\frac{u}{U}\right) e^{C_{2} x}, \quad Y_{\eta}\left(\frac{u}{U}\right) e^{C_{4} x} \leqslant \frac{u_{y y}}{u_{y}} \leqslant Y_{\eta}\left(\frac{u}{U}\right) e^{-C_{5} x}, \\
\exp \left[-\frac{M_{1}^{2}}{4} y^{2} e^{2 C_{2} x}\right] \leqslant 1-\frac{u}{U} \leqslant \exp \left[-\frac{M_{1}^{2}}{4} y^{2} e^{-2 C_{1} x}\right] .
\end{gathered}
$$

где $Y(\eta)$ - решение задачи (6), (7).

4. Вспомогательные утверждения. Доказательство существования решения задачи (4), (5) проведем на основе метода прямых, т.е. дискретизацией по $\xi$ и заменой уравнения (4) системой обыкновенных дифференциальных уравнений.

Для любой функции $f(\tau, \xi, \eta)$ введем обозначение

$$
\begin{gathered}
f^{m, k}=f^{m, k}(\eta) \equiv f(m h, k h, \eta), \quad h=\text { const }>0, \\
m=1,2, \ldots, \quad k=0,1, \ldots,[X / h], \quad 0 \leqslant \eta \leqslant 1 .
\end{gathered}
$$

Уравнения (4) с условиями (5) заменим системой уравнений

$$
\begin{aligned}
& L_{m, k}(w):=\nu(1+\left.3 d(k h)^{2}\left(V^{m, k}\right)^{2}\left(w^{m, k}\right)^{2}\right)\left(w^{m, k}\right)^{2} w_{\eta \eta}^{m, k}-\frac{w^{m, k}-w^{m-1, k}}{h}- \\
&-\eta k h V^{m, k} \frac{w^{m, k}-w^{m, k-1}}{h}+\left(\eta^{2}-1\right)\left(V^{m, k}+k h V_{\xi}^{m, k}\right) w_{\eta}^{m, k}- \\
&-\eta\left(V^{m, k}+k h V_{\xi}^{m, k}\right) w^{m, k}+6 \nu d(k h)^{2}\left(V^{m, k}\right)^{2}\left(w^{m, k}\right)^{3}\left(w_{\eta}^{m, k}\right)^{2}=0
\end{aligned}
$$

с граничными условиями

$$
\begin{gathered}
w^{0, k}(\eta)=w_{0}(m h, \eta), \quad w^{m, k}(1)=0, \\
l_{m, k}(w):=\left.\left(\nu w^{m, k} w_{\eta}^{m, k}\left(1+3 d(k h)^{2}\left(V^{m, k}\right)^{2}\left(w^{m, k}\right)^{2}\right)-v_{0}^{m, k} w^{m, k}+C^{m, k}\right)\right|_{\eta=0}=0 .
\end{gathered}
$$

Далее всюду $C_{i}, M_{i}$ - положительные постоянные, не зависящие от $h$.

Доказательство основных результатов проводим с помощью следующих лемм.

Лемма 1. Предположим, что при $0 \leqslant x \leqslant X$ выполнено неравенство $U_{x}>0 ;$ функиии $V, V_{x}$, $v_{0}$ ограничены,

$$
\left|\frac{V_{t}}{V}\right| \leqslant M_{1} x, \quad M_{2}(1-\eta) \leqslant w_{0}(\xi, \eta) \leqslant M_{3}(1-\eta)
$$


$u w_{0}(\xi, \eta)$ имеет непрерывную производную по $\eta \in[0,1)$. Тогда при $0 \leqslant m h \leqslant \infty, 0 \leqslant k h \leqslant X$, где $X$ зависит от $V, v_{0}, w_{0}$, задача (8), (9) имеет решение $w^{m, k}(\eta)$, обладающее непрерывной производной третъего порядка при $0 \leqslant \eta<1$. Для этого решения справедливы неравенства

$$
C_{1}(1-\eta) \leqslant w^{m, k}(\eta) \leqslant C_{2}(1-\eta) .
$$

Доказательство леммы проводится методом эллиптической регуляризации.

Лемма 2. Пусть выполнены условия леммы 1 и функиия $v_{0 x}(t, x)$ ограничена при $0 \leqslant t \leqslant \infty$ $u 0 \leqslant x \leqslant X$. Пусть, кроме того, выполнены неравенства

$$
Y(\eta) e^{-M_{4} \xi} \leqslant w_{0}(\xi, \eta) \leqslant Y(\eta) e^{M_{5} \xi} .
$$

Тогда при $0 \leqslant m h \leqslant \infty, 0 \leqslant k h \leqslant X$, где $X$ зависит от $V, v_{0}$, решения $w^{m, k}(\eta)$ задачи (8), (9) удовлетворяют неравенствам

$$
Y(\eta) e^{-C_{3} k h} \leqslant w^{m, k}(\eta) \leqslant Y(\eta) e^{C_{4} k h} .
$$

Лемма 3. Пусть выполнены условия леммы 2 и, кроме того,

$$
\begin{gathered}
\left|w_{0 \xi}\right| \leqslant M_{6}(1-\eta), \quad Y_{\eta} e^{M_{7} \xi} \leqslant w_{0 \eta} \leqslant Y_{\eta} e^{-M_{8} \xi}, \\
-M_{9}(1-\eta) \leqslant \nu\left(1+3 d \xi^{2} V^{2} w_{0}^{2}\right) w_{0}^{2} w_{0 \eta \eta}+A w_{0 \eta}+B w_{0}+6 \nu d \xi^{2} V^{2} w_{0}^{3} w_{0 \eta}^{2} \leqslant M_{10} \xi(1-\eta), \\
\left.\left(\nu\left(1+3 d \xi V^{2} w_{0}^{2}\right) w_{0} w_{0 \eta}-v_{0} w_{0}+C\right)\right|_{\eta=0, \tau=0}=0,
\end{gathered}
$$

где $A_{\xi}, B_{\xi}, C_{\xi}$ ограниченъ,

$$
\left|V_{\tau}\right| \leqslant M_{11} \xi, \quad B_{\tau} \leqslant M_{12} \xi, \quad C_{\tau} \leqslant M_{13} \xi, \quad\left|v_{0}\right| \leqslant M_{14}, \quad v_{0 \tau} \leqslant-M_{15} \xi .
$$

Тогда при $0 \leqslant m h \leqslant \infty, 0 \leqslant k h \leqslant X$, где $X$ зависит от $V, v_{0}$, решения $w^{m, k}(\eta)$ задачи (8), (9) удовлетворяют неравенствам

$$
\begin{gathered}
-C_{5}(1-\eta) \leqslant \frac{w^{m, k}-w^{m-1, k}}{h} \leqslant C_{6} k h(1-\eta), \quad\left|\frac{w^{m, k}-w^{m, k-1}}{h}\right| \leqslant C_{7} Y, \quad k \geqslant 1, \\
Y_{\eta}(\eta) e^{C_{8} k h} \leqslant w_{\eta}^{m, k}(\eta) \leqslant Y_{\eta}(\eta) e^{C_{9} k h}, \quad\left|w^{m, k} w_{\eta \eta}^{m, k}\right| \leqslant C_{10}, \quad w^{m, k} w_{\eta \eta}^{m, k} \leqslant-C_{11},
\end{gathered}
$$

где постоянные $C_{j}$ не зависят от $h$.

Автор выражает глубокую признательность проф. В. Н. Самохину и своему научному руководителю проф. Г. А. Чечкину за постановку задачи и ценные советы при планировании исследования и рекомендации по оформлению статьи.

\section{СПИСОК ЛИТЕРАТУРЫ}

1. Булатова Р. Р., Самохин В. Н., Чечкин Г. А. Уравнения магнитогидродинамического пограничного слоя для модифицированной несжимаемой вязкой среды. Отрыв пограничного слоя// Пробл. мат. анал. - 2018. - 92. - C. 83-100.

2. Олейник O. А., Самохин В. Н. Математические методы в теории пограничного слоя. - М.: Наука, 1997.

3. Самохин В. Н., Фадеева Г. М., Чечкин Г. А. Уравнения пограничного слоя для модифицированной системы Навье-Стокса// Тр. семин. им. И. Г. Петровского. - 2011. - 28. - С. 329-361.

4. Самохин В. Н., Чечкин Г. А. Уравнения пограничного слоя обобщенно ньютоновской среды в окрестности критической точки// Тр. семин. им. И. Г. Петровского. - 2016. - 31. - С. 158-176.

5. Bulatova R. R., Chechkin G. A., Chechkina T. P. and Samokhin V. N. On the influence of a magnetic field on the separation of the boundary layer of a non-Newtonian MHD medium// C. R. Mecanique. — 2018. 346, № 9. - P. 807-814.

Булатова Регина Рашидовна

Московский государственный университет им. М. В. Ломоносова

E-mail: regina.bulatova@mech.math.msu.su 